\title{
Construcción y caracterización de un detector de cámara proporcional multialambres
}

\section{Construction and characterization of a multiwire proportional chamber detector}

\author{
M.J. Delgado González ${ }^{\text {a* }}$ \\ H. F. Castro Serrato ${ }^{a}$
}

Fecha de recepción: 07.08.18

Fecha de aceptación: 19.03.2019

DOI: https://doi.org/10.19053/01217488.v10.n2.2019.8304

\begin{abstract}
Resumen
Se construyó y caracterizó un detector gaseoso de partículas tipo "cámara proporcional multialambres", conocida por sus siglas en inglés como MWPC. El detector consta de un circuito electrónico dentro de una caja hermética de acrílico en una atmósfera de gas apropiado. Una vez verificado su funcionamiento mecánico y eléctrico, se realizó la caracterización como detector de partículas. Se midió la curva característica, consistente en la carga eléctrica colectada en los electrodos en función del voltaje aplicado. Luego se midió la curva de conteo de muones provenientes de la radiación cósmica en función del voltaje, conocida como "curva Plateau". Las mediciones se realizaron con diferentes mezclas de gases: $100 \%$ Aire, $100 \% \mathrm{Ar}, 100 \% \mathrm{CH} 4$ y $90 \% \mathrm{Ar}+10 \% \mathrm{CH} 4$ (P10). En los resultados se discute el funcionamiento del detector para distintos voltajes, con estas mezclas de gas. El mejor desempeño fue obtenido con la mezcla P10, para la cual el detector funciona en la región proporcional, para voltajes entre $3700 \mathrm{~V}$ y 4000 $\mathrm{V}$, con un voltaje óptimo de operación de $3850 \mathrm{~V}$.
\end{abstract}

Palabras clave: Detectores de partículas, cámara proporcional multialambres, cámara de ionización, contador proporcional, curva característica.

a Grupo CRYOMAG, Departamento de Física, Universidad Nacional de Colombia, 11001 Bogotá, Colombia.

* Correo electrónico: mjdelgadog@unal.edu.co, hfcastros@unal.edu.co 


\begin{abstract}
We have built and characterized a gaseous particle detector of the type "multiwire proportional chamber", known as MWPC. The detector consists of an electronic circuit inside a hermetically sealed acrylic box, in an appropriate atmosphere of gas. Once the mechanical and electrical operation was verified, we performed the characterization as a particle detector. We measured the characteristic curve, consisting of the electrical charge collected at the electrodes, as function of applied voltage. Then we measured the count rate, for muons from cosmic radiation, versus voltage, known as "Plateau curve". These measurements were performed with different mixtures of gases: $100 \% \mathrm{Air}, 100 \% \mathrm{Ar}, 100 \% \mathrm{CH} 4$ and $90 \%$ $\mathrm{Ar}+10 \% \mathrm{CH} 4(\mathrm{P} 10)$. In the results we discuss the operation of the detector at different voltages, for these gas mixtures. The best performance was obtained with the P10 mixture, for which, the detector works in the proportional region, for voltages between $3700 \mathrm{~V}$ to $4000 \mathrm{~V}$, with an optimal operating voltage of $3850 \mathrm{~V}$.
\end{abstract}

Key words: Particle detectors, multiwire proportional chamber, ionization chamber, proportional counter, characteristic curve.

\section{INTRODUCCIÓN}

Entre los años 1967 y 1968 se desarrolló el detector gaseoso "cámara proporcional de multialambres" (MWPC), por el físico Georges Charpak (1968). Este dispositivo sentó nuevos precedentes en el campo de los detectores de partículas, por su capacidad de localización, mejora en la resolución espacial de unos cientos de micras, y excelente resolución energética. Además, la cámara MWPC permite registrar hasta un millón de conteos por segundo. Este dispositivo inició el camino hacia la creación de detectores con mayor velocidad, precisión $\mathrm{y}$, tal vez lo más importante, la digitalización de las señales para su integración a un sistema computarizado.

La MWPC contribuyó en la realización de experimentos, los cuales permitieron, entre otras: encontrar nuevas partículas masivas, descubrir los bosones W y Z en el CERN (Knoll 1989), el quark charm en SLAC y Brookhaven y el "quark top" en el Fermilab (Sauli 1978). Actualmente, este dispositivo continúa siendo un componente esencial de investigación en algunos experimentos como el LHC en el CERN (ALICE collaboration 2008) y OLYMPUS en Alemania (Milner 2014), en detectores de neutrones (Wang 2018) y otras aplicaciones en física de altas energías y física médica (Sharma 2001).

Es así que la cámara MWPC constituye un hito en el desarrollo de los detectores de partículas, por los beneficios y logros que se alcanzaron con su invención. Por ello se considera importante el estudio de este detector, como un primer paso en la implementación de un laboratorio de investigación de detectores gaseosos. La motivación del grupo CRYOMAG para la realización de este trabajo, es la función didáctica que representa el acercamiento práctico a la fabricación y caracterización de un detector gaseoso. El objetivo a mediano plazo es incursionar en la investigación de detectores gaseosos para diferentes aplicaciones y la participación del grupo en la colaboración internacional RD 51 del CERN, la cual busca el desarrollo tecnológico de los detectores gaseosos y sus aplicaciones(Pinto 2010).

Este trabajo es la versión resumida de la Tesis de Maestría de Maritza Delgado (2014).

\section{FUNDAMENTOS TEÓRICOS}

\subsection{Física de los detectores gaseosos}

Un detector gaseoso consta de un par de electrodos (ánodo y cátodo) conectados a un alto voltaje en una atmósfera de gas apropiado. La radiación que incide sobre el gas genera ionizaciones, denominadas ionizaciones primarias, como se observa en la figura 1 . Los iones y electrones generados fluyen hacia el electrodo correspondiente, por acción del campo eléctrico. Los electrones al ser acelerados pueden, a su vez, generar ionizaciones secundarias y procesos de avalancha, logrando así un efecto de multiplicación (Sauli 1977). La carga total colectada en los electrodos constituye la señal del 
detector, la cual debe ser procesada (amplificación, discriminación, integración, conteo, etc.), a fin de obtener la información deseada.

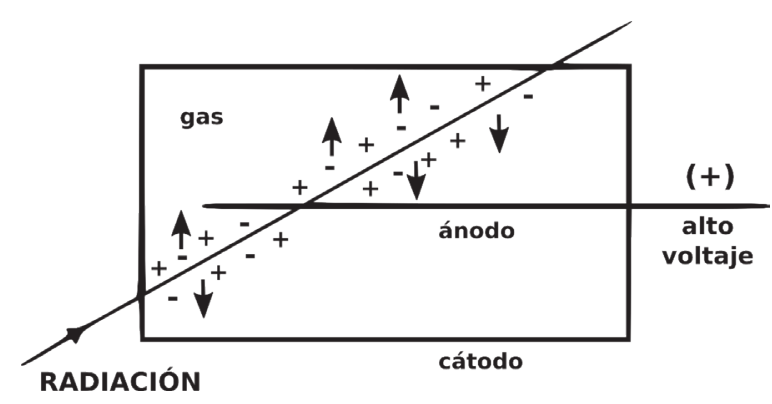

Figure 1: Principio básico de operación de un detector gaseoso. La radiación incidente ioniza el gas y las cargas generadas ،fluyen hacia los electrodos, por la acción del campo eléctrico, donde son colectadas formando la señal de detección.

\section{Avalanchas y multiplicación}

El efecto de multiplicación en un detector se basa en la formación de avalanchas, las cuales dependen de la intensidad del campo eléctrico. Para un detector de alambres las avalanchas se producen muy cerca al ánodo y se asemejan a gotas, con un frente ancho de electrones y una larga cola que se va estrechando, formada por una nube de iones positivos que se desplazan lentamente hacia el cátodo.

\section{Sofocación o "Quench” y selección del gas}

Los electrones acelerados por el campo eléctrico no solamente producen ionizaciones secundarias; también pueden causar excitación de los átomos del gas, los cuales al desexcitarse emiten fotones, usualmente en el rango UV. Al interactuar estos fotones con las paredes internas del detector, el ánodo o el cátodo, pueden arrancar electrones por efecto fotoeléctrico. Estos electrones también serán atraídos por el ánodo dando origen a nuevas avalanchas, cuyo origen no guarda correlación directa con la trayectoria de la partícula detectada, por lo cual se pierde la capacidad de rastreo. Por otro lado, se puede llegar a saturar todo el volumen del detector, reduciendo así la tasa de conteo. Por ello es deseable eliminar los fotones UV mediante un gas poliatómico, aprovechando la gran cantidad de estados excitados no radiactivos, o grados internos de libertad de las moléculas orgánicas, los cuales permiten la absorción de fotones en un gran rango de energía. Este proceso de eliminación de fotones, o sofocamiento de la avalancha, es conocido en inglés como quench. El metano, en particular, cubre el rango de energía de los fotones emitidos por el Argón (Charpak 1968).

En principio, un detector gaseoso podría operar con cualquier gas; sin embargo, se elige uno con el fin de optimizar varios parámetros importantes, como: bajo voltaje de operación, alta ganancia, alta movilidad de iones, largo tiempo de vida útil y rápida recuperación, entre otros. (Flakus 1981).

Se pre"eren los gases nobles con baja energía de ionización, ya que debido a su baja reactividad química se reducen los efectos de deterioro de los electrodos y las paredes internas del detector. Dadas las consideraciones anteriores, lo más recomendable es la mezcla de un gas noble, como el argón o el xenón, con un gas poliatómico, como el metano o el dióxido de carbono.

\subsection{Curva característica de respuesta}

La curva característica de un detector es una gráfica en la que se presenta el número de Carga, o iones, colectados en función del voltaje entre los electrodos. La curva característica presenta diferentes zonas o regímenes de trabajo relacionados con la dinámica de las cargas dentro del detector y los procesos de avalancha y multiplicación. Estas dependen, principalmente, de la geometría de los electrodos y del gas de trabajo. La curva está dividida en las siguientes cinco zonas: 1. Zona de recombinación, 2. Cámara de ionización, 3. Contador proporcional, 4. Zona de proporcionalidad limitada, 5. Contador Geiger-Muller. Para una descripción detallada de la curva característica y los modos de operación de un detector gaseoso ver (Knoll 1989).

\subsection{Cámara Proporcional Multialambres MWPC}

Los contadores proporcionales son utilizados para medir la energía depositada por la radiación, pero adolecen de baja capacidad de localización espacial, limitando su función a la determinación 
del paso de la partícula por el detector. A fin de remediar esta deficiencia, Charpack, desarrolló la MWPC en 1967-1968, la cual consiste de un conjunto de alambres equidistantes, muy próximos entre sí, conectados al mismo potencial (ánodo); y uno, o dos planos catódicos, conectados a tierra. De esta manera, cada alambre actúa como un contador independiente, permitiendo la determinación espacial del punto de cruce de la partícula detectada.

En la figura 2 se ilustra la geometría de la MWPC y las líneas de campo eléctrico en su interior. Los electrones se desplazan a lo largo de las líneas de campo, hacia los ánodos, muy cerca de los cuales se produce la multiplicación por avalanchas (Knoll 1989). Por su parte, los iones positivos se desplazan en la dirección contraria, hacia los planos catódicos.

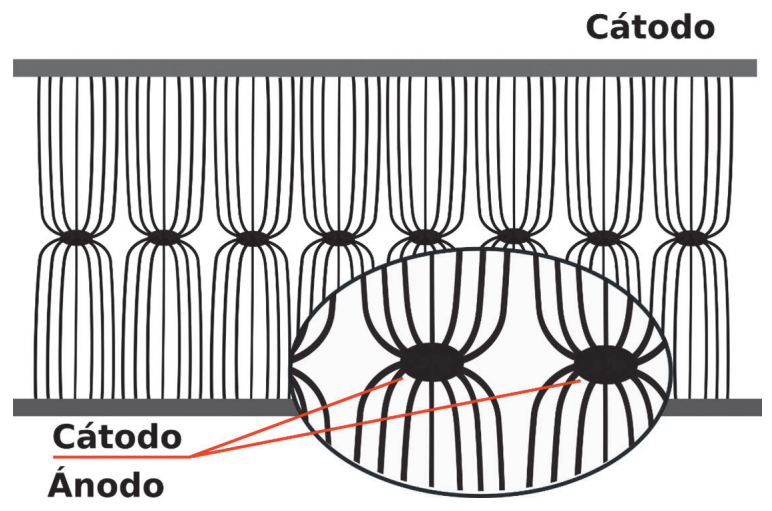

Figure 2: Estructura básica y líneas de campo eléctrico en la MWPC [2]. El campo eléctrico se intensifica en la cercanía de los ánodos, donde ocurren las avalanchas y efecto de multiplicación. En el resto del volumen el campo eléctrico es bastante uniforme.

\section{MATERIALES Y MÉTODOS}

\subsection{Construcción de la Cámara MWPC}

Se construyó el prototipo con base en los criterios y parámetros reportados por Alfaro (2005) y Sauli (1978). Los ánodos se hicieron de alambre de tungsteno recubierto en oro, por ser buen conductor eléctrico y resistente al bombardeo de partículas (Grupen 2008); el recubrimiento en oro impide la oxidación. La longitud de los alambres fue de $50 \mathrm{~mm}$ y diámetro de $25 \mu \mathrm{m}$. El espaciamiento entre ellos se tomó en $5 \mathrm{~mm}$, a fin de facilitar la realización de los puntos de soldadura en el soporte de baquelita.

La figura 3 (derecha) muestra una foto del circuito electrónico, conformado por una placa de baquelita de $100 \mathrm{~mm} \times 100 \mathrm{~mm} \times 1 \mathrm{~mm}$, con una ventana de $65 \mathrm{~mm} \times 35 \mathrm{~mm}$, sobre la cual se soldaron 12 alambres. El cátodo consiste en una segunda placa de baquelita recubierta de cobre, ubicada a $4 \mathrm{~mm}$, debajo del plano de los alambres, soportada por cuatro espaciadores de teflón.

El detector se colocó dentro de una caja hermética de acrílico transparente, en una atmósfera del gas de trabajo. Las dimensiones externas de la caja son $200 \times 200 \times 50 \mathrm{~mm}^{3}$, la tapa superior se fija con tornillos y se sella con una junta redonda de goma. La caja contiene dos racores, uno para la entrada y el otro para la salida del gas. En la caja se instalaron también los conectores de alto voltaje y salida de la señal, como se ilustra en la figura 3 (izquierda).

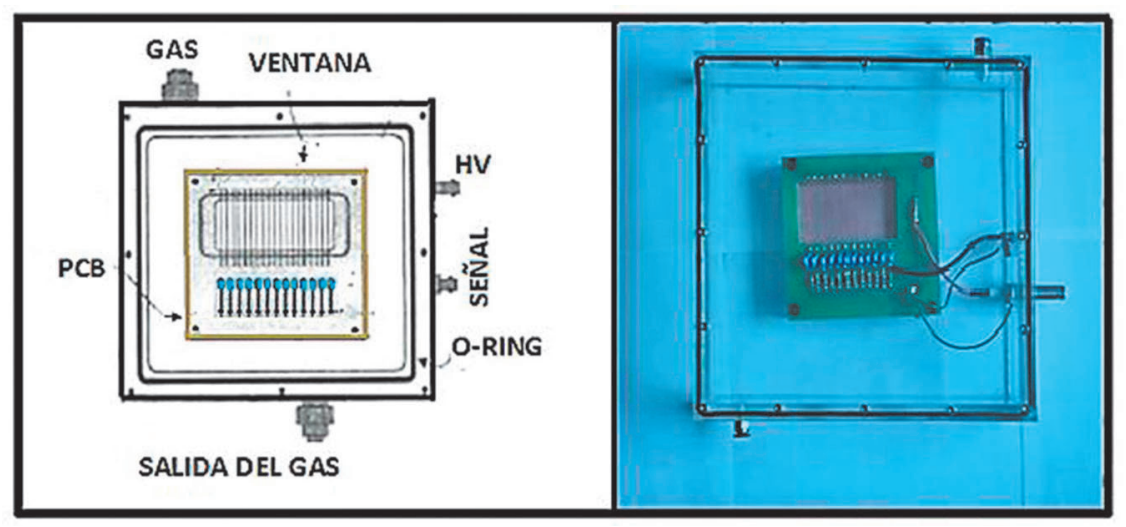

Figure 3: Detalle de la Cámara MWPC construida. Diagrama (izquierda), con el detector dentro de la caja hermética de acrílico, la cual contiene 2 conexiones para el flujo de gas, alimentación de alto voltaje (HV) y salida de la señal eléctrica. Circuito electrónico (derecha), conformado por 12 alambres conectados a la línea de alto voltaje mediante una resistencia $\mathrm{R}(1.5 \mathrm{M} \Omega$ ). Del otro extremo se conectan los alambres a un condensador y una resistencia a tierra. La señal de salida se toma de los condensadores. El cátodo es la placa inferior de baquelita recubierta de cobre, la cual se conecta a tierra. 
El circuito eléctrico de polarización y lectura de la señal, para cada ánodo, se ilustra en la "gura 4. Cada ánodo es conectado a un condensador $\mathrm{C}$ de $220 \mathrm{pF}$, a fin de desacoplar la señal del alto voltaje; este va en serie con una resistencia $r$, de $50 \Omega$, que acopla la impedancia del detector con la entrada de los módulos electrónicos. La fuente de alto voltaje se conecta a los ánodos por el otro extremo, a través de una resistencia $\mathrm{R}$ de 1.5 $\mathrm{M} \Omega$, a fin de limitar la corriente, en caso de chispas. Para la caracterización del detector los condensadores de los 12 alambres se conectaron en paralelo, sumando así las señales de todos los ánodos en una sola salida.

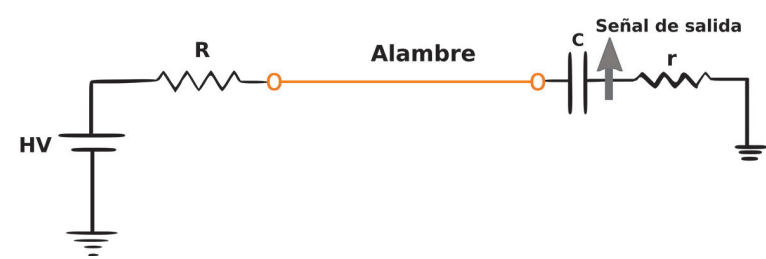

Figure 4: Circuito eléctrico de polarización y lectura. Cada ánodo se encuentra conectado a una resistencia $(\mathrm{r}=50)$ y un condensador de desacople $(\mathrm{c}=220 \mathrm{pF})$. La fuente de alto voltaje se conecta al otro extremo del alambre, mediante una resistencia $\mathrm{R}=1.5 \mathrm{M}$, para controlar la corriente, en caso de chispas.

\subsection{Gas de llenado}

Se eligió como gas principal el argón, ya que es el más utilizado en los detectores gaseosos por ser un gas noble, de fácil ionización y bajo costo.

Por otro lado, se utilizó aire y metano, a modo de comparación. Como gas de sofocamiento, se eligió el metano, ya que cubre el rango de energía de los fotones emitidos por el argón (Sauli 1977).

Finalmente, se usó la mezcla $90 \% \mathrm{Ar}+10 \%$ CH4 conocida como P10, la cual es de uso frecuente, en particular en cámaras proporcionales (Alfaro 2005).

\subsection{Fuente de radiación}

A falta de una fuente radiactiva de suficiente actividad, se usaron los muones cósmicos, como fuente de radiación, lo cual es frecuente en la caracterización de detectores (Sauli 1978). Estos presentan un alto nivel de penetración y a su vez de interacción con el gas, generando así una señal eléctrica adecuada (Sharma 2001).
El flujo de muones cósmicos sobre la tierra está muy bien caracterizado (Grupen 2011), y se conoce que a nivel del mar es de (1 muón)/ $\left(\mathrm{cm}^{2} \times \min \right)$. Este flujo presenta una pequeña variación para ángulos polares inferiores a 45 grados, sin embargo esta variación fue ignorada en este trabajo tomando el valor de flujo arriba anotado.

\subsection{Montaje Experimental}

El montaje para la caracterización del detector (figura 5), consta de un soporte metálico de instrumentos con módulos electrónicos de procesamiento de pulsos, tablero de control de flujo de gas con flujometros de esfera, tanques de gas y un soporte para el detector. Los módulos electrónicos utilizados (NIM) incluyen: amplificador CAEN N914, contador Camberra 512, discriminador CAEN N841, analizador multicanal CAEN N957, dos fuentes duales de alto voltaje Ortec $556 \mathrm{H}$ y osciloscopio digital LeCroy Wavesurfer MXS-B.

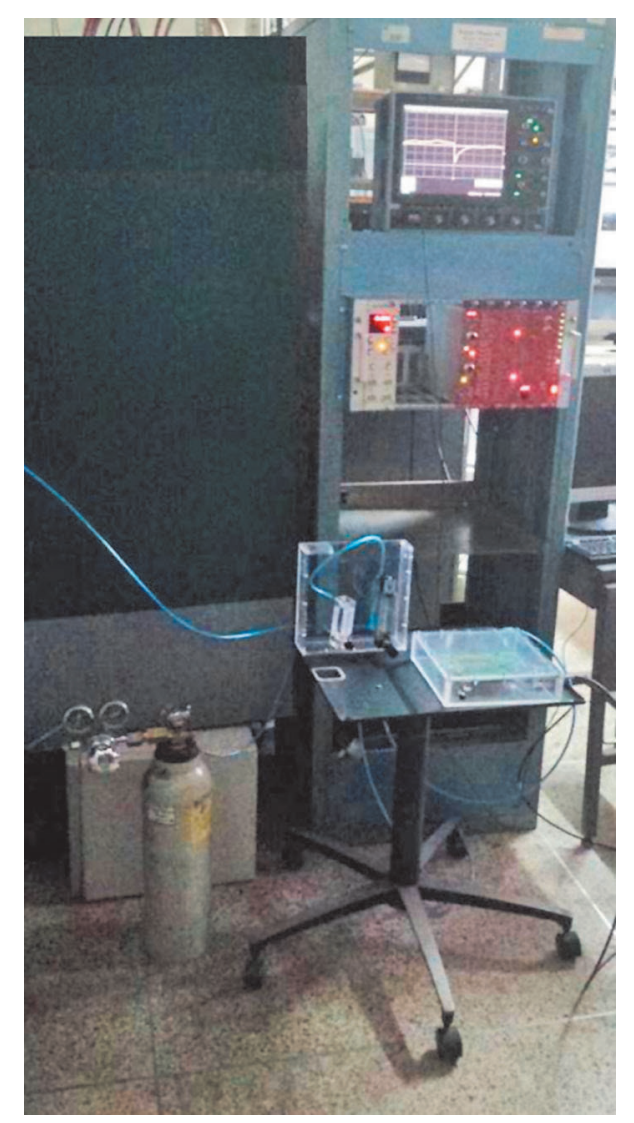

Figure 5: Montaje de caracterización del detector. Conformado por un soporte metálico de instrumentos, tablero de control de flujo de gas, soporte del detector y tanques de gas. 


\section{RESULTADOS Y DISCUSIÓN}

Primero, se realizaron pruebas del circuito electrónico y sus conexiones de entrada (HV) y salida (señal). Posteriormente se probó la hermeticidad de la caja de acrílico y flujo del gas. Para las mediciones de caracterización la cámara fue operada con un flujo constante de gas de 2 $\mathrm{L} / \mathrm{h}$. Antes de iniciar cada medición, se dejó fluir el gas, aproximadamente 10 minutos, a presión ligeramente superior a la atmosférica.

Todas las mediciones se realizaron con la configuración del circuito ilustrado en la figura 6. El bloque de instrumentación cambia, según el tipo de medición a realizar, como se explica más adelante. Se caracterizó la cámara MWPC, tomando una sola señal de los 12 alambres, conectando las 12 salidas en paralelo.

El primer paso consistió en estudiar cualitativamente los pulsos generados por el detector y determinar el rango de voltajes de operación sin chispas, para cada mezcla de gas. En segundo lugar se midió la curva característica, para lo cual se registraron directamente los pulsos mediante el osciloscopio, promediando 100 muestras para cada voltaje. Se calculó el número de iones colectados integrando numéricamente los pulsos medidos, al no contar con la electrónica suficiente . Luego se midió la tasa de conteo de muones en función del voltaje, obteniendo la curva de Plateau. Se realizaron mediciones con las mezclas de gas: 100\% aire, 100\% Argón (Ar), $100 \%$ metano $(\mathrm{CH} 4)$, y $90 \% \mathrm{Ar}+10 \% \mathrm{CH} 4$ (P10). A continuación se describe cada uno de estos procedimientos y sus resultados.

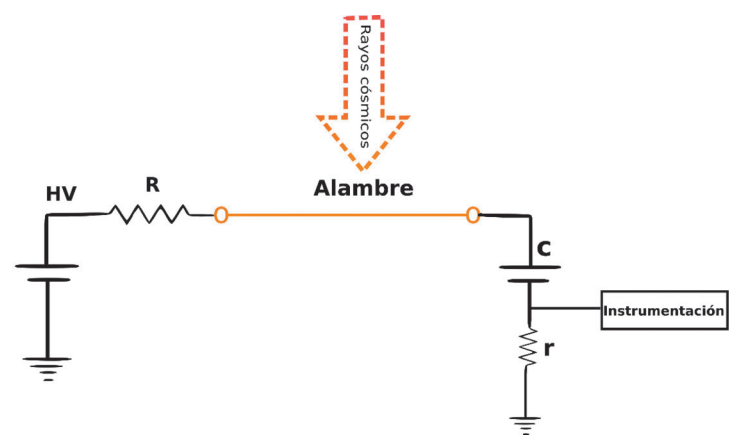

Figure 6: Circuito de medición. El bloque de instrumentación depende del tipo particular de medición a realizar.

\subsection{Análisis de pulsos y rango de voltajes de operación}

Se analizaron los pulsos adquiridos directamente con el osciloscopio. Para esta medición, en la figura 6 el bloque de instrumentación consiste únicamente del osciloscopio. Los pulsos obtenidos fueron todos de polaridad negativa. Al aumentar el voltaje se observa una variación de la altura y la forma de los pulsos y al sobrepasar el voltaje máximo de operación, los pulsos se deforman. Los rangos de voltaje de operación, sin chispas, se resumen en el cuadro 1.

Table 1: Rangos de voltaje de operación para cada mezcla de gas Mezcla de Gas.

\begin{tabular}{cc}
\hline Mezcla de Gas & $\begin{array}{c}\text { Voltaje de } \\
\text { operación (V) }\end{array}$ \\
\hline Argón & $1400-2400$ \\
Aire & $2850-4600$ \\
Metano & $2200-2800$ \\
P10 & $2600-4000$ \\
\hline
\end{tabular}

\subsection{Curva Característica}

Para esta medición, el bloque de instrumentación del circuito de la figura 6, consiste en un amplificador (Ö10) seguido del osciloscopio. Se registró el promedio de 100 muestras en el osciloscopio, y para cada voltaje se calculó el número de cargas colectadas utilizando la ecuación:

$$
Q=\frac{A}{r e}
$$

donde A es el área bajo la curva del pulso, $r$ la resistencia $(50 \Omega)$ y e la carga del electrón. Los pulsos se analizaron "off-line" integrando numérica-mente para hallar el área, mediante un código de programación en $\mathrm{C}++$, el cual permitió mayor precisión. La figura 7 muestra los resultados obtenidos. 

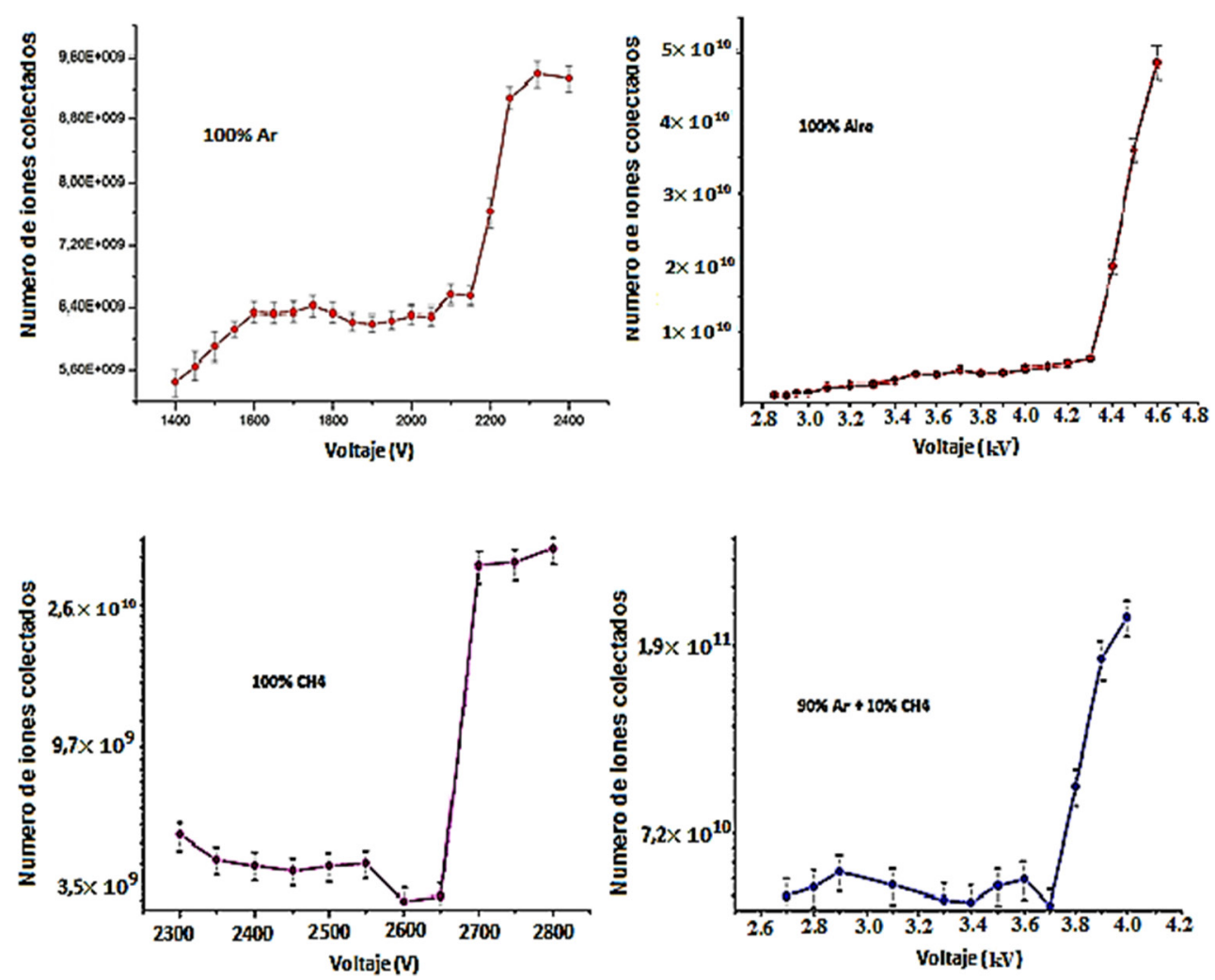

Figure 7: Curva característica. Se pueden observar los rangos de voltaje en los que opera la MWPC, con diferentes tipos de gas. Se tiene el mínimo de voltaje de operación con 100\% Ar en 1400 V, el máximo voltaje de operación con aire a $4600 \mathrm{~V}$ y máxima ganancia $\sim 10^{11}$, para la mezcla P-10.

\section{Análisis}

\section{Argón 100\%}

Se observan pulsos desde $1400 \mathrm{~V}$, y a $3000 \mathrm{~V}$ se presentan chispas. Entre $1400 \mathrm{~V}$ y $1600 \mathrm{~V}$ hay un pequeño aumento de la carga colectada, indicando que se está en la región de recombinación. Entre $1600 \mathrm{~V}$ a $2100 \mathrm{~V}$, la carga no cambia notablemente con el voltaje, correspondiente a la región de cámara de ionización. A partir de $2200 \mathrm{~V}$ se observa un aumento abrupto en la carga colectada comportándose de modo proporcional. (Curva naranja).

\section{Aire}

Se obtienen pulsos a partir de $3000 \mathrm{~V}$, y al llegar a $4650 \mathrm{~V}$ se presentan chispas. Para voltajes menores a $4400 \mathrm{~V}$, hay un incremento suave de la carga, indicando la región cámara de ionización. A partir de $4400 \mathrm{~V}$, se observa un comportamiento de contador proporcional. Aunque el aire requiere mayor voltaje para generar multiplicación, puede servir como gas proporcional bajo circunstancias especiales, como se discute en Sauli (1978). (Curva roja).

\section{Metano 100\%}

Se observan pulsos a partir de $2200 \mathrm{~V}$, y al llegar a $2900 \mathrm{~V}$ se presentan chispas. De $2200 \mathrm{~V}$ hasta $2600 \mathrm{~V}$, el numero de cargas colectadas no cambia notablemente, correspondiente a la región de cámara de ionización. Para voltajes mayores a $2650 \mathrm{~V}$, se presenta un incremento abrupto de la carga colectada, asociado probablemente al modo de operación Streamer. Entre 2700 V y 2800 $\mathrm{V}$ se presenta una corta meseta de estabilidad. Aunque el Metano es más utilizado como gas de sofocación (quencher), también es utilizado como gas proporcional, en espectroscopia de neutrones, a pesar de requerir mayores voltajes de operación (Sauli 1977). (Curva morada)

\section{Mezcla P10 (90\% Ar + 10\% CH4)}

Se obtienen pulsos a partir de $2200 \mathrm{~V}$, y al llegar a $4050 \mathrm{~V}$ se presentan chispas. La carga colectada entre $2600 \mathrm{~V}$ y $3600 \mathrm{~V}$ no varía mucho, indicando una operación como cámara de ionización. Entre $3700 \mathrm{~V}$ y $4000 \mathrm{~V}$, la carga aumenta linealmente entrando a funcionar en la zona proporcional. (Curva azul). 
La mezcla P10, en comparación con las mezclas anteriores y con otras mezclas como $82 \% \mathrm{Ar}+18 \% \mathrm{CO}_{2}($ Varga 2015$)$ y $70 \% \mathrm{Ar}+$ $30 \% \mathrm{CO}_{2}$ (Patra 2016), presenta un mayor rango de voltaje de operación, mayor ganancia, buen funcionamiento por más tiempo y operación en modo proporcional.

\subsection{Curva de Conteo Vs. Voltaje o curva Plateau}

Para esta medición se utilizó el circuito de la figura 6 , compuesto por un amplificador $(\times 10)$
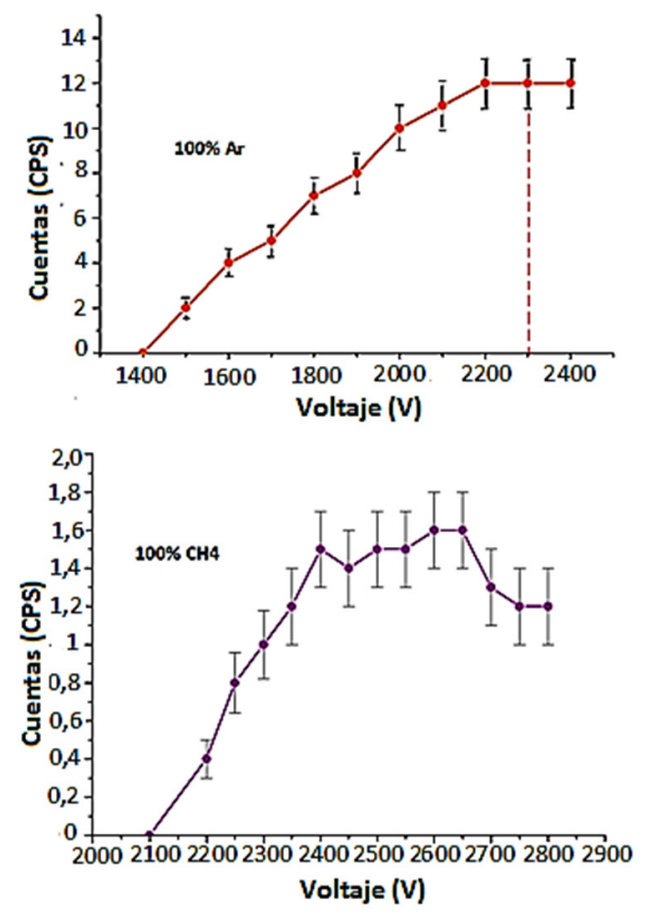

seguido de un discriminador y luego el contador. Antes de iniciar el conteo se ajusto el valor de discriminación 2ara cada mezcla, entre $120 \mathrm{mV}$ y $250 \mathrm{mV}$, a fin de disminuir el ruido electrónico y de fondo. El conteo se realizó en intervalos de tiempo de $10 \mathrm{~s}, 30 \mathrm{~s}$ o $60 \mathrm{~s}$, dependiendo de la mezcla utilizada.

Se tomaron 3 datos para cada voltaje y se calculó el promedio, en cuentas por segundo (CPS). La figura 8 presenta estos resultados.
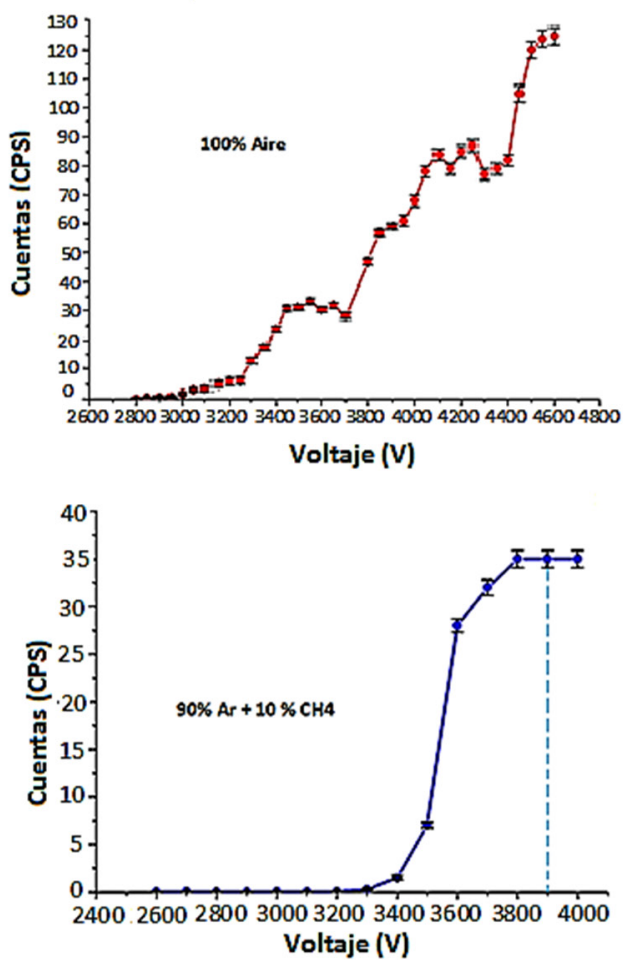

Figure 8: Cuentas por segundo en función del voltaje o curva plateau. De estas curvas se determina el voltaje óptimo de operación (VOP), como el 25\% del Plateau [9]. El aire presenta un comportamiento errático, sin punto estable de operación.

En el cuadro 2 se resumen los parámetros que caracterizan la curva de conteo para cada tipo de gas. Se encontró un mayor conteo para el aire y un menor conteo para el metano.

Table 2: Voltajes óptimos de operación (VOP) y conteo máximo.

\begin{tabular}{ccc}
\hline Gas & Conteo máximo (CPS) & $\begin{array}{c}\text { Voltaje óptimo de } \\
\text { operación }(\mathbf{V})\end{array}$ \\
\hline Ar & $12 \pm 1.1$ & 2250 \\
Aire & $125 \pm 2$ & - \\
CH4 & $1.2 \pm 0.2$ & 2725 \\
P10 & $35 \pm 8$ & 3850 \\
\hline
\end{tabular}




\section{CONCLUSIONES}

Se diseñó y construyó un detector tipo Cámara Proporcional de Multialambres (MWPC). Se Caracterizó con cuatro tipos de gases: 100\% aire, $100 \%$ Argón (Ar), 100\% metano (CH4) y la mezcla P10 (90\% $\mathrm{Ar}+10 \% \mathrm{CH} 4)$.

El detector se caracterizó determinando: el rango de voltaje de operación (desde la aparición de pulsos hasta la generación de chispas), curva característica (cargas colectadas vs. Voltaje) y curva de conteo vs. Voltaje (curva plateau). De las curvas características se determinó el modo de operación de la cámara, mientras que de la curva plateau se halló el voltaje óptimo de operación.

Los mejores resultados se obtuvieron con la Mezcla P10 (90\% Ar + 10\% CH4), entre los intervalos de voltaje $2600 \mathrm{~V}-4000 \mathrm{~V}$. Para la mezcla P10, se observó una zona de cámara de ionización entre $2600 \mathrm{~V}$ a $3600 \mathrm{~V}$. Para voltajes de $3700 \mathrm{~V}$ a $4000 \mathrm{~V}$, la cámara MWPC funciona en la zona proporcional, alcanzando una ganancia máxima de $\sim 2 \times 10^{11}$.

\section{AGRADECIMIENTOS}

Los autores agradecen al Departamento Administrativo de Ciencia, Tecnología e Innovación - COLCIENCIAS, la financiación parcial de este proyecto.

\section{REFERENCES}

[1] G. Charpak; et. al. The Use of Multiwire Proportional Chambers to Select and Localize Charged Particles. Nucl. Instr. Meth. 62:262268, 1968.

[2] G.F Knoll. Radiation Detection and Measurement. New York, Estados Unidos: J. Wiley\&Sons, 1989.

[3] F. Sauli. Principles of operation of multiwire proportional and drift chambers, yellow report. CERN 77-09,1977.
[4] R. Patra; R. Singaraju. Time resolution and characteristic studies of MWPC Detectors with Argon Based Gas Mixtures. Springer proceedings in physics. 203:509-512, 2018.

[5] C. Grupen; B. Shwartz. Particle Detectors, Cambridge University Press. Cambridge: Segunda edición, 2011.

[6] S. Tavernier. Experimental Tecniques in nuclear and Particle Physics. Berlin, Heidelberg, Alemania: Springer, 2010.

[7] F. Sauli . Limiting accuracies in multiwire proportional and drift chambers, Nucl. Instr. Meth. 156: 147-157, 1978.

[8] F.N. Flakus. Detección y medición de las radiaciones ionizantes: historia sucinta. OIEA Boletin. Vol. 23 No. 4: 31-36, 1981.

[9] A. Sharma. Gaseous Micropattern Detectors: High-Energy Physics and Beyond. ICFA Instrum. Bull:22, 2001.

[10] R. Alfaro. Construction and operation of a small Multiware proportional chamber. Journal of Physics. 18:362, 2005.

[11] S.D. Pinto. Micropattern gas detector technologies and applications: The work of the RD51 collaboration. Bonn, Germany: Physikalisches Institut der Universitat Bonn, 2010.

[12] D. Abriola; A.C Pichel; P. Galagovsky. Caracterización de un detector de muones, ANALES ALFA. 18, 2006.

[13] W. Blum; L. Rolandi. Particle detection with drift chambers. Berlin, Heidelberg, Alemania: Springer, 1993.

[14] W.R. Leo. Techniques for Nuclear and Particle Physics Experiments. Berlin, Heidelberg, Alemania: Springer, 1987.

[15] M. J. Delgado. Diseño y construcción de una cámara proporcional de Multialambres, Tesis de Maestría, Departamento de Física, Universidad Nacional de Colombia, Bogotá, Colombia, 2014. 
[16] M. Ghahremani; S. Ashra"; J. Rahighi. Basic design of a multiwire proportional counter using Gar"eld++ for ILSF. Journal of Instrumentation. V1.11 No. 12: 120-121, 2016.

[17] Z. Ahmeda; M. A. Bowlesb; R. Bunkerb. A prototype low-background multiwire proportional chamber for measuring alphas and low-energy betas. Journal of Instrumentation. Vol.9 No. 1, 2014.

[18] CERN Photo Archive, The ALICE Time Projection Chamber: journey from building 2252 to the ALICE cavern, ALICE-PICTTPC-2007-003, 2007. https://cds.cern.ch/ record/1011033.

[19] M. Titov; L. Ropelewski. Micro-pattern gaseous detector technologies and RD51 Collaboration. Modern Physics Letters. Vol.28, No.13, 1340022, 2013.

[20] D. Varga; Z. Gal. Cosmic Muón Detector Using Proportional Chambers. European Journal of Physics. 36,2015.
[21] J. Pan; C. Jan. Development of a portable and fast wire tension measurement system for MWPC construction. Chinese Physics. Vol.40, No 9, 096201, 2016.

[22] R. Patra; R. Singaraju. Study of a Multiware Proportional Chambers (MWPC) detector using NIM and MANAS based electronics. Proceedings of the DAE-BRNS on Nucl. Phys. 61, 2016.

[23] H. Wang; Y. Zhao. Prototype of neutron sensitive MWPC detector with delay-line readout. Nuclear Techniques.Vol.41, No 8, 2018.

[24] R. Milner; D. Hasell. The OLYMPUS experiment. Nuclear Instruments and Methods in Physics Research. 741: 1-17, 2014.

[25] ALICE collaboration. The ALICE experiment at the CERN LHC. JINST. Vol.3, 2008. 